\title{
LARGE MINIMAL REALIZERS OF A PARTIAL ORDER
}

\author{
STEPHEN B. MAURER ${ }^{1}$ AND I. RABINOVITCH
}

\begin{abstract}
The size of a minimum realizer of a partial order is called the dimension of that partial order. Here we initiate the study of minimal realizers which are not minimum. As an aid to the study of such realizers, we associate to each minimal realizer certain critical digraphs. We characterize all such critical digraphs for the antichain on $n$ elements, and consequently deduce that for $n>4$, the maximum size of a minimal realizer is $\left[n^{2} / 4\right]$.
\end{abstract}

A partially ordered set or poset, $(X, P)$, consists of a set $X$ together with an irreflexive and transitive relation $P$ on it. If $(a, b)$ is in the relation, we say that $a$ is above or over $b$. In this paper $X$ will always be a finite set. A realizer of $P$ is a set of linear orders $\mathcal{L}=\left\{L_{1}, L_{2}, \ldots, L_{r}\right\}$ whose intersection is $P$. A minimal realizer of $P$ is one which does not contain as a proper subset another realizer of $P$. A minimum realizer is one of minimum size. The number of elements in a minimum realizer of $P$ is the dimension of $P$, a concept which has been studied by a number of authors in recent years. See [4] for a good bibliography on dimension. In this paper we shift the focus to minimal realizers which are not necessarily of minimum size, and in particular look at minimal realizers of maximum size, henceforth to be called maximin realizers. We define the rank of $P$ to be the number of elements in a maximin realizer of $P$.

Although the dimension of posets has received considerable attention recently, it remains extremely difficult to find the dimension of an arbitrary poset $\boldsymbol{P}$. However, it is much easier to find a minimal realizer of $\boldsymbol{P}$. Starting with any realizer $\mathcal{L}=\left\{L_{1}, L_{2}, \ldots, L_{r}\right\}$, take the intersection $\bigcap_{i \neq 1} L_{i}$. If it equals $P$ then $e-L_{1}$ is a realizer of $P$. If not, try casting out $L_{2}$, and so on until a minimal realizer is obtained. The number of elements in this realizer of $P$ can be considered an approximation to the dimension of $P$. Subsequent results in this paper will show that this first approximation need not be very good at all, thus indicating the need for a more sophisticated approach to approximating the dimension of an arbitrary poset.

Let $\mathcal{L}=\left\{L_{1}, L_{2}, \ldots, L_{r}\right\}$ be a realizer of $P$. If $(b, a) \in L_{j}$, and $(a, b) \in L_{i}$ for all $i \neq j$, we say that $L_{j}$ is critical for the ordered pair $(a, b)$.

Received by the editors January 26, 1977.

AMS (MOS) subject classifications (1970). Primary 06A05, $05 \mathrm{C} 20$.

Key words and phrases. Linear order, antichain, realizer, digraph, acyclic, unipathic, partial order.

${ }^{1}$ Work supported by National Science Foundation Grant MCS76-19670.

- American Mathematical Society 1977 
LemMa 1. $\mathcal{E}=\left\{L_{1}, L_{2}, \ldots, L_{r}\right\}$ is a minimal realizer of $P$ if and only if each $L_{i}$ is critical for some ordered pair of elements of $X$.

Proof. Suppose $L_{k}$ is not critical for any ordered pair. Then $\bigcap_{i \neq k} L_{i}=P$ and $\mathcal{E}$ is not minimal. To prove the converse, suppose each $L_{i}$ is critical for some ordered pair $\left(a_{i}, b_{i}\right)$. Then $\bigcap_{i \neq k} L_{i}$ strictly contains $P$ since $\left(a_{k}, b_{k}\right) \notin P$ but $\left(a_{k}, b_{k}\right) \in L_{i}$ for each $i \neq k$.

The above lemma states only that each member of a minimal realizer is critical for at least one ordered pair. It is possible, as the reader may easily see by means of an example, for a linear order to be critical for more than one ordered pair. From each $L_{i} \in \mathcal{L}$, choose one ordered pair for which it is critical. Let $D(P, \mathcal{E})$, or simply $D$, be the set of all these chosen ordered pairs. Then $(X, D)$ is an irreflexive and (if $|\mathcal{E}| \geqslant 3$ ) asymmetric digraph which we will call a critical graph of $(P, \mathcal{L})$. We say a critical graph rather than the critical graph since in general there will be many different graphs arising from $(P, \mathcal{L})$ in this way.

Up to this point we have not assumed anything special about $P$. We now concentrate on the case when $P$ is the antichain $A_{n}$ on $n$ elements, i.e., the partial order where no element is over another. We will use critical graphs to describe all minimal realizers of $A_{n}$. In particular, we will determine the rank of $A_{n}$.

First, one more definition: a digraph is unipathic if, for each pair of vertices $a, b$, there is at most one directed path from $a$ to $b$, and at most one from $b$ to $a$. As is natural when associating digraphs with irreflexive binary relations, we do not consider a vertex to be connected to itself by a null path. Thus a unipathic digraph may have cycles. (A cycle in a digraph is understood to be directed, although this will sometimes be made explicit.) Henceforth we shall use AU as an abbreviation for acyclic and unipathic. The following lemma is required in our first theorem. A proof may be found in [2].

Lemma 2. Let $C$ be a chain of a partially ordered set $(X, P)$. Then the transitive closure of

$$
P \cup\{(x, c): c \text { is in } C \text { and } x \text { is not below } c\}
$$

is a partial order on $X$.

THeOREM 3. Let $(X, P)$ be an antichain with $|X| \geqslant 2$, let $\mathcal{E}$ be a minimal realizer of $P$, and let $(X, D)$ be a critical graph of $(P, \mathcal{L})$. Then $(X, D)$ is either (1) an acyclic-unipathic digraph, or (2) a directed cycle.

Proof. Suppose $D$ contains a directed cycle and at least one edge $(x, y)$ is not in the cycle. Therefore, there is at least one order $L$ in the realizer, namely the one containing $(y, x)$, which contains every edge in the cycle, a contradiction.

Suppose now that there are (at least) two directed paths from some $s$ to some $t$. Without loss of generality we may suppose the paths are edge-

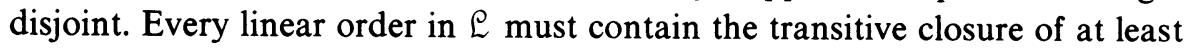


one of these paths. This means that $(s, t)$ is an element of every linear order in $\mathcal{L}$ and therefore is in their intersection, contradicting the assumption that $\mathcal{L}$ is a realizer of an antichain.

We now prove the converse.

TheOREM 4. Let $D$ be a digraph with vertex set $X$ and at least two edges. Then $D$ is a critical graph for some minimal realizer of the antichain on $X$ if either

(1) $D$ is a directed cycle, or

(2) $D$ is acyclic and unipathic.

Proof. For $(x, y) \in D$, define $D(x, y)=D \cup(y, x)-(x, y) . D(x, y)$ can be thought of as being obtained from $D$ by reversing the orientation of the edge $(x, y)$. Let $D^{t}(x, y)$ be the transitive closure of $D(x, y)$. Since $D$ is unipathic in either case 1 or $2, D(x, y)$ must be acyclic and hence $D^{t}(x, y)$ is a partial order. Next, let $L(x, y)$ be any linear order which (i) extends $D^{t}(x, y)$ and which (ii) has the property that every element not below $x$ (resp. $y$ ) in $D^{t}(x, y)$ is above $x$ (resp. $y$ ) in $L(x, y)$. It is a consequence of Lemma 2 that such an $L(x, y)$ exists.

By considering cases we will show that for any ordered pair $(b, a)$ there is some $(x, y) \in D$ such that $(b, a) \in L(x, y)$. Thus, if we set $\mathcal{L}=\{L(x, y)$ : $(x, y) \in D\}$, we have that $\cap \mathcal{E}$ is the antichain. Furthermore, it is obvious that $\mathcal{L}$ is then a minimal realizer of the antichain with critical graph $D$, because $L(x, y)$ is critical for $(x, y)$ in $\mathcal{L}$.

For now, assume that the graph $D$ has no isolated vertices and is of type 2 .

CASE I. There is a path from $a$ to $b$ in $D$. Let $x$ be the vertex after $a$ in this path (perhaps $x=b)$. Since the only path in $D$ from $a$ to $b$ uses $(a, x)$, there can be no path from $a$ to $b$ in $D(a, x)$. Thus $b$ is not below $a$ in $D^{t}(a, x)$, so $(b, a) \in L(a, x)$.

CASE II. There is no path from $a$ to $b$ in $D$ and $a$ has positive outdegree. There is some $x$ such that $(a, x) \in D$, and clearly there is no path from $a$ to $b$ in $D(a, x)$. Thus $b$ is not below $a$ in $D^{t}(a, x)$.

CASE III. Vertex $a$ has zero outdegree. Since we assume no vertex is isolated, we may pick $x$ such that $(x, a) \in D$.

CASE IIIA. $x=b$. Since $|D| \geqslant 2$, for any $(y, z) \in D$ other than $(b, a)$, we have $(b, a) \in L(y, z)$.

CASE IIIB. $x \neq b$ and there is no path from $x$ to $b$. Then there is no path from $a$ to $b$ in $D(x, a)$ because $(a, x)$ is the only edge out of $a$ in $D(x, a)$. Thus $(b, a) \in L(x, a)$.

CASE IIIC. $x \neq b$ and there is a path from $x$ to $b$. Let $y$ be the vertex after $x$ in this path. By the same argument as in Case $\mathrm{I}$, we conclude that $(b, x) \in L(x, y)$. Since $(x, a) \in L(x, y)$, by transitivity $(b, a) \in L(x, y)$.

We have proved the theorem for AU graphs without isolated vertices. Now let $D$ be a cycle, still without isolated vertices. Since for any pair of vertices $a$, $b$, there is a path from $a$ to $b$, Case I above is the only case. The proof given 
for Case I is valid without change for cycle graphs.

Finally, let $D$ be either type 1 or 2 , and suppose there is a nonempty set $Y$ of isolated vertices. We modify our construction of the $L(x, y)$ slightly to insure that each $(b, a)$ is in some $L(x, y)$ even when $a$ is in $Y$. Pick a fixed linear order $L^{\prime}$ on $Y$ and for all $(x, y) \in D$ except one, call it $\left(x^{\prime}, y^{\prime}\right)$, insist that $L(x, y)$ extend $L^{\prime}$ as well as meet the old conditions (i) and (ii). It is always possible to find such an $L(x, y)$, since the transitive closure $D^{\prime}$ of $D(x, y) \cup L^{\prime}$ is a partial order and any element which is not below $x$ (resp. $y$ ) in $D^{t}(x, y)$ is not below $x$ (resp. $y$ ) in $D^{\prime}$ either; thus Lemma 2 still applies. As for $L\left(x^{\prime}, y^{\prime}\right)$, choose it so that all elements of $Y$ are below everything else, so that its restriction to $Y$ is the reverse of $L^{\prime}$, and so that otherwise it still satisfies (i) and (ii); it is easy to show that this is always possible. Since $|D| \geqslant 2, \cap \mathcal{E}$ is the antichain, $\mathcal{E}$ is a minimal realizer, and $D$ is a critical graph for $\mathcal{L}$.

In order to determine the rank of the antichain $A_{n}$, all that is left is to make one observation and recall one theorem of graph theory. The observation is this: there are just 2 distinct orientations of a triangle; one is a cycle and the other contains 2 paths between the same 2 points. Hence no acyclic, unipathic digraph contains a triangle. Second, a well-known theorem of Turan [6] states that each triangleless graph on $n$ vertices (directed or not) contains at most $\left[n^{2} / 4\right]$ edges and that this bound is obtained only for the complete bipartite graph with $[n / 2]$ vertices on one side and $\{n / 2\}$ on the other. $[k]$ and $\{k\}$ denote the greatest integer less than or equal to $k$, and least integer greater than or equal to $k$, respectively.

THEOREM 5. The rank of the antichain $A_{n}$ is $\max \left(n,\left[n^{2} / 4\right]\right)$.

Proof. By Theorems 3 and 4, it suffices to compare the number of edges in the biggest cycle on $n$ vertices with the number of edges in the biggest AU digraph on $n$ vertices. The former number is $n$. In view of the previous paragraph, we will know that the latter number is $\left[n^{2} / 4\right]$ as soon as we verify that a complete bipartite graph has an AU orientation. But this is easy-direct all edges from one side to the other. Finally, $\left[n^{2} / 4\right] \geqslant n$ iff $n \geqslant 4$, with equality only when $n=4$.

One can actually say a bit more about the maximin realizers of $A_{n}$. Let $K$ be the complete bipartite graph with $[n / 2]$ vertices on one side and $\{n / 2\}$ on the other. If $n \geqslant 4$, the only way to direct $K$ so as to make it AU is to direct it from one side to the other, or vice versa; these are distinct situations when $n$ is odd. There are no other AU orientations because if a 4-cycle is to be AU, it cannot contain a directed path of length 2 .

One can also show that if $\mathcal{L}$ is a maximin realizer of $A_{n}$, where $n$ is arbitrary, then $\mathcal{E}$ has just one critical graph. In other words, each $L \in \mathcal{E}$ is critical for just one pair $(x, y)$. We omit the (routine) demonstration of this assertion.

Since the dimension of the antichain $A_{n}$ is 2 for all $n \geqslant 2$, we have an 
example of our claim that the size of a minimal realizer may be a very poor approximation to the size of a minimum realizer. There are other examples. For instance, let $P(j, k, n)$ be the partial order of set inclusion on all $j$-subsets and all $k$-subsets of an $n$-set, where $1 \leqslant j<k \leqslant n-1$. The dimension of $P(j, k, n)$ is at most $n$, since the dimension of the boolean algebra of all subsets of an $n$-set is $n$ [3]. However, as we will show elsewhere [5], the rank of $P(j, k, n)$ is exactly $\left(\begin{array}{l}n \\ k\end{array}\right)\left(\begin{array}{l}n \\ j\end{array}\right)-\left(\begin{array}{c}n \\ k\end{array}\right)\left(\begin{array}{l}k \\ j\end{array}\right)$. For the case $j=1, k=n-1$, this expression equals $n$. For all other permissible values of $j$ and $k$, the value of this expression is strictly greater than $n$. Since the dimension of $P(1, n-1, n)$ is $n$ [3], we can see that it is the only $P(j, k, n)$ whose dimension is equal to its rank.

Finally, the following question seems interesting in its own right. What undirected graphs can be given AU orientations? We have noted that they must be triangleless, but this is not sufficient. To see this, first note that any orientation at all of an odd cycle must contain a path of length 2. As was mentioned earlier, an AU orientation of a 4-cycle cannot contain a path of length 2; indeed, there is essentially just one AU orientation of a 4-cycle. Thus Figure 1, a circular ladder of odd length, does not have an AU orientation; since each 4-cycle must be $\mathrm{AU}$, the rungs must alternately point in and out, which is impossible. We thank Bruce Hedman for this example.

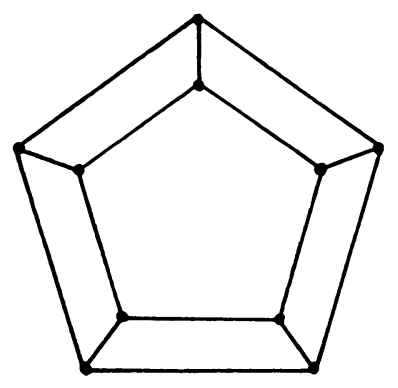

Figure 1

We have discussed AU orientations with V. Chvátal. He has proved (personal communication) that the problem of determining whether an arbitrary graph has an AU orientation is NP-complete; see [1] for a discussion of this important concept in the theory of algorithmic complexity. Chvátal's result still leaves open the possibility of finding broad and useful necessary conditions or sufficient conditions for a graph to have an AU orientation.

\section{REFERENCES}

1. A. V. Aho, J. E. Hopcroft and J. D. Ullman, The design and analysis of computer algorithms, Addison-Wesley, Reading, Mass., 1974.

2. K. P. Bogart, Maximal dimensional partially ordered sets. I, Hiraguchi's theorem, Discrete Math. 5 (1973), 21-31. 
3. H. Komm, On the dimension of partially ordered sets, Amer. J. Math. 70 (1948), 507-526.

4. W. T. Trotter, Jr. and J. I. Moore, Jr., Some theorems on graphs and posets, Discrete Math. 15 (1976), $79-84$.

5. S. Maurer and I. Rabinovitch, Large minimal realizers of a partial order. II (submitted).

6. P. Turan, An extremal problem in graph theory, Math. Lapok. 48 (1941), 536-552.

Department of Mathematics, Princeton University, Princeton, New Jersey 08540

Department of Mathematics, Rutgers University, New Brunswick, New Jersey 08903 